\title{
Levetiracetam in the treatment of epilepsy
}

\author{
Bassel Abou-Khalil \\ Department of Neurology, Vanderbilt \\ University Medical Center, Nashville, \\ Tennessee, USA
}

\begin{abstract}
Epilepsy is a common chronic disorder that requires long-term antiepileptic drug therapy. Approximately one half of patients fail the initial antiepileptic drug and about 35\% are refractory to medical therapy, highlighting the continued need for more effective and better tolerated drugs. Levetiracetam is an antiepileptic drug marketed since 2000. Its novel mechanism of action is modulation of synaptic neurotransmitter release through binding to the synaptic vesicle protein SV2A in the brain. Its pharmacokinetic advantages include rapid and almost complete absorption, minimal insignificant binding to plasma protein, absence of enzyme induction, absence of interactions with other drugs, and partial metabolism outside the liver. The availability of an intravenous preparation is yet another advantage. It has been demonstrated effective as adjunctive therapy for refractory partial-onset seizures, primary generalized tonic-clonic seizures, and myoclonic seizures of juvenile myoclonic epilepsy. In addition, it was found equivalent to controlled release carbamazepine as first-line therapy for partial-onset seizures, both in efficacy and tolerability. Its main adverse effects in randomized adjunctive trials in adults have been somnolence, asthenia, infection, and dizziness. In children, the behavioral adverse effects of hostility and nervousness were also noted. Levetiracetam is an important addition to the treatment of epilepsy.
\end{abstract}

Keywords: epilepsy, seizures, antiepileptic drugs, long-term therapy, efficacy, safety, levetiracetam

\section{Introduction - long-term management considerations in epilepsy}

Epilepsy is a chronic condition characterized by recurrent unprovoked epileptic seizures. Epileptic seizures are the clinical manifestations including symptoms and signs of an abnormal, excessive, and hypersynchronous electrical discharge of neurons in the brain. Thus, a seizure is a symptom. Epilepsy is a condition; it cannot be considered a disease because it can be caused by many etiologies. Epilepsy may be genetic or could be the result of a variety of insults to the brain, including head trauma, stroke, vascular malformations, or congenital brain malformations (Engel 2001). Because seizures and epilepsy are very heterogeneous they have to be classified. The most widely used classification is that proposed by the International League Against Epilepsy in 1981, dividing seizures into those that are partial and those that are generalized (Commission 1981). Partial seizures are ones in which the first clinical and electrographic changes suggest initial activation limited to part of one cerebral hemisphere. Partial seizures are further subdivided into simple partial, complex partial and partial becoming generalized. Simple partial seizures are those in which awareness and responsiveness are completely preserved. Complex partial seizures involve at least an alteration of responsiveness or awareness. Secondarily generalized seizures can start either as simple partial or complex partial, but then spread to the whole brain and most often manifest towards their later part with generalized tonic and then clonic activity. Generalized seizures are those in which the first clinical changes indicate initial involvement of both hemispheres. Consciousness is usually impaired at onset, except for myoclonic 
seizures which are too brief for altered consciousness to be appreciated. Motor manifestations are bilateral if they occur. The initial electrographic ictal patterns are bilateral. Generalized seizure types include generalized absence, generalized myoclonic, generalized tonic, generalized clonic, generalized tonic clonic, and generalized atonic seizures.

In addition to the classification of epileptic seizures, the International League Against Epilepsy proposed a classification of epilepsies and epileptic syndromes (Commission 1981, 1989). Since most patients have either partial seizure types or generalized seizure types, the two main subdivisions in the classification are partial (focal, local, or localizationrelated) epilepsies, and generalized epilepsies. Each of these major categories is sub-classified into those epilepsies that are idiopathic and presumed genetic or symptomatic/cryptogenic (probably symptomatic), related to a brain insult. In general, idiopathic epilepsies respond better to treatment than symptomatic epilepsies. Within this epilepsy classification are epileptic syndromes that are characterized by a specific range of age at onset, specific seizure types, specific natural history or course, and specific response to treatment. For example, juvenile myoclonic epilepsy is a type of idiopathic generalized epilepsy in which patients have generalized myoclonic seizures, particularly after awakening, generalized tonic clonic seizures (in about 90\%), and generalized absence seizures (in about $30 \%$ of cases). In this syndrome, the electroencephalogram (EEG) shows generalized 4-6 Hz spike-and-wave discharges in between seizures. These patients respond well to treatment but their epilepsy is a lifelong condition (Renganathan and Delanty 2003). Some forms of epilepsy are known to have a limited course, with remission expected. For example, benign childhood epilepsy with centrotemporal spikes, also called benign rolandic epilepsy, is an epileptic syndrome in which seizures are usually infrequent, easily controlled, and remit at puberty (Wirrell 1998). However, most epilepsies are chronic and require long-term therapy.

The treatment of epilepsy will depend on appropriate classification of the seizure type and the epileptic syndrome, then the choice of an antiepileptic drug (AED) that is most appropriate for the seizure type and epileptic syndrome and also the safest and most appropriate for the patient's particular medical background. The treatment of epilepsy should always begin with monotherapy, using a low initial dose and titrating slowly. Among the more than sixteen marketed antiepileptic drugs approximately one half are older agents marketed before 1980, while the rest were marketed after 1990 (Table 1) (Schachter 2007). The older
AEDs were generally approved for marketing and even used as first-line agents without undergoing the rigorous clinical trials now required of the newer antiepileptic drugs. Regulatory approval for the new AEDs is restricted to the specific epilepsy patient populations in whom the drug has demonstrated efficacy and to the specific mode of use in the relevant clinical trial. For example, a new AED will receive approval for first-line monotherapy use only if demonstrated effective as first-line monotherapy in a sound clinical trial. If the new AED is not started as first-line monotherapy, but monotherapy is achieved after removal of an existing AED, then the regulatory approval will be for conversion to monotherapy only. Among the newer AEDs, the vast majority were initially tested and approved for use as adjunctive therapy. Monotherapy trials typically followed later. Such trials have earned several AEDs approval for monotherapy use. However, the regulatory agencies are not uniform in their criteria for approval of AED indications: some agents have been approved for monotherapy in Europe but not in the US.

If seizures continue despite maximum tolerated doses of the first AED, a change in therapy is indicated. Although an alternative monotherapy is usually recommended at this point, there is no scientific evidence to support the strategy of alternative monotherapy over adjunctive therapy (Kwan and Brodie 2000b; Beghi et al 2003). In general, common sense would decree that if the first drug is not tolerated or if it is totally ineffective, alternative monotherapy is the best approach. If the first drug was well tolerated and was at least partially effective, adjunctive therapy could be considered. The choice of first alternative monotherapy or add-on therapy depends on several factors, including safety, tolerability, efficacy in clinical trials, ease of use, potential for rapid titration, pharmacokinetic interactions, efficacy in co-morbidities, and less prominently mechanism of action. If adjunctive therapy is chosen, potential interactions between the first and the second AED are important factors in the choice of AED (Patsalos and Perucca 2003). Patients who fail a second AED are much less likely to become seizure free with the third next AED than those who have failed only one AED (Kwan and Brodie 2000a). After failure of two or three AEDs, patients with partial epilepsy should be considered for epilepsy surgery, which is highly effective in certain "surgically remediable" epileptic syndromes such as temporal lobe epilepsy with hippocampal sclerosis or focal epilepsy associated with certain benign brain lesions. Patients who are not excellent candidates for epilepsy surgery can undergo additional AED trials, including AED 
Table I Spectrum of efficacy of standard (A), and new AEDs (B). The new AEDs are listed in the order of their marketing in the US, following approval by the US Food and Drug Administration

\begin{tabular}{|c|c|c|c|c|c|}
\hline & & Partial & I ary GTC & G myoclonic & G absence \\
\hline \multirow[t]{8}{*}{ A } & Phenytoin & + & + & - & - \\
\hline & Carbamazepine & + & + & - & - \\
\hline & Valproate & + & + & + & + \\
\hline & Phenobarbital & + & + & - & - \\
\hline & Primidone & + & + & + & - \\
\hline & Ethosuximide & - & - & - & + \\
\hline & Methsuximide & + & $?$ & $?$ & + \\
\hline & Clonazepam & + & + & + & + \\
\hline \multirow[t]{9}{*}{ B } & Felbamate & $+^{\mathrm{b}}$ & + & $?$ & $?$ \\
\hline & Gabapentin $^{\mathrm{a}}$ & $+^{\mathrm{b}}$ & - & - & - \\
\hline & Lamotrigine $^{\mathrm{a}}$ & $+^{\mathrm{b}}$ & $+^{\mathrm{b}}$ & $?$ & $+^{\mathrm{b}}$ \\
\hline & Topiramate $^{\mathrm{a}}$ & $+^{\mathrm{b}}$ & $+^{\mathrm{b}}$ & $?$ & $?$ \\
\hline & Tiagabine & $+^{b}$ & $?$ & - & - \\
\hline & Oxcarbazepine ${ }^{a}$ & $+^{\mathrm{b}}$ & $+?$ & - & - \\
\hline & Levetiracetam $^{\mathrm{a}}$ & $+^{*}$ & $+^{\mathrm{b}}$ & $+{ }^{\mathrm{b}}$ & $?$ \\
\hline & Zonisamide & $+^{\mathrm{b}}$ & + & + & $?$ \\
\hline & Pregabalin & $+^{\mathrm{b}}$ & $?$ & - & - \\
\hline
\end{tabular}

aNew AED with positive initial monotherapy trials.

bNew AED efficacy indication supported by blinded trials.

combinations. In general it is advisable to avoid combinations of more than three AEDs because of the risk of interactions and additive adverse effects. Non-pharmacological therapies such as vagus nerve stimulation and the ketogenic diet or modified Atkins diet can also be considered in patients who fail to respond to or are unable to tolerate antiepileptic drugs. However, vagus nerve stimulation is unlikely to produce seizure freedom, and compliance with the ketogenic or Atkins diet can be a major challenge.

Even though the landmark study of Kwan and Brodie suggested that the chances of seizure freedom with a new AED decrease with the failure of each additional AED, one survey of patients who failed epilepsy surgery evaluation found that $21 \%$ had achieved seizure remission at follow up, most often due to the addition of one of the new AEDs (Selwa et al 2003). Levetiracetam, the focus of this review is one of these new AEDs.

\section{Levetiracetam}

Levetiracetam (LEV) is one of the newest AEDs, marketed worldwide only since 2000 . It was initially approved in the US only as adjunctive therapy for partial-onset seizures. However, more recent trials earned it approval as adjunctive therapy for primary generalized tonic-clonic seizures and myoclonic seizures of juvenile myoclonic epilepsy, and a recent comparative monotherapy trial earned it approval for use as initial monotherapy in the European Union, though not in the US. In addition, the recent approval and marketing of an intravenous preparation has added to the versatility of this AED.

\section{Levetiracetam pharmacology}

LEV is rapidly and almost completely absorbed after oral intake, with peak plasma concentrations approximately one hour after oral administration. Food reduces the peak plasma concentration by $20 \%$ and delays it by 1.5 hours, but does not reduce LEV bioavailability (Patsalos 2000, 2003). There is a linear relationship between LEV dose and LEV serum level over a dose range of 500-5000 mg (Radtke 2001). LEV protein binding, at less than $10 \%$, is not clinically relevant. LEV metabolism is not dependent on the liver cytochrome P450 enzyme system. LEV is predominantly excreted unchanged through the kidneys, with only about $27 \%$ metabolized. The main metabolic pathway is hydrolysis of the acetamide group in the blood (Radtke 2001). The resultant metabolite generated is inactive. LEV plasma half-life is $7 \pm 1$ hours in adults, but can be prolonged by an average of 2.5 hours in the elderly, most likely due to decreased creatinine clearance with age (French 2001; Hirsch et al 2007). In patients with impaired renal function, a dose adjustment is needed, dependent on the creatinine clearance (French 2001). The absence of hepatic metabolism and of protein binding predict absence of pharmacokinetic interactions (Nicolas et al 1999). Indeed, no pharmacokinetic interactions were observed with phenytoin, warfarin, digoxin, or oral contraceptives (Browne et al 2000; Levy et al 2001; Patsalos 2000, 2003; 
Ragueneau-Majlessi et al 2001, 2002; Abou-Khalil et al 2003; Coupez et al 2003). However, some studies have suggested lower LEV levels or higher LEV clearance in patients taking enzyme-inducing AEDs (May et al 2003; Perucca et al 2003; Hirsch et al 2007). Autoinduction probably does not occur with LEV, but one study involving short intensive monitoring suggested a drop in serum levels after the fifth day of administration (Stefan et al 2006).

\section{Intravenous levetiracetam}

The intravenous formulation of LEV was demonstrated bioequivalent to the oral formulation (Ramael et al 2006b). In the initial study $1,500 \mathrm{mg}$ of LEV were injected over 15 minutes (Ramael et al 2006b). The infusion was well tolerated and adverse effects were similar to those with oral LEV, though somnolence was more common with the intravenous administration. In a second study, higher doses and faster infusion rates were used $(2,000,3,000$, and 4,000 mg over $15 \mathrm{~min} ; 1,500,2,000$, and 2,500 $\mathrm{mg}$ over $5 \mathrm{~min}$ ) (Ramael et al 2006a). The most common adverse experiences, dizziness and somnolence, were not clearly related to dose or infusion rate. As expected, the peak plasma level was reached at 5 or 15 minutes, corresponding to the end of the infusion, but otherwise the pharmacokinetic profile was similar to that of oral LEV. LEV infusion over 15 minutes was demonstrated to be a practical alternative in epilepsy patients unable to take the oral medication (Baulac et al 2007).

\section{Pharmacology in children, infants, and neonates}

Pharmacokinetics in children were studied in 15 boys and nine girls 6-12 years old who received a single dose of LEV, $20 \mathrm{mg} / \mathrm{kg}$ as an adjunct to their stable regimen of a single concomitant AED (Pellock et al 2001). The half-life was $6 \pm 1.1$ hours. The C-max and area under the curve were lower in children than in adults and renal clearance was higher. The apparent body clearance was $1.43 \pm 0.36 \mathrm{~mL} / \mathrm{min} / \mathrm{kg}$, $30 \%-40 \%$ higher in children than in adults. In another study in younger children and infants, the same dose/Kg was administered as a $10 \%$ oral solution to thirteen subjects aged 2.3-46.2 months. The mean half-life was $5.3 \pm 1.3$ hours in this younger group (Glauser et al 2007). The half-life is likely longer in neonates. Two studies estimated LEV halflife in the neonate at 18 hours (Allegaert et al 2006; Tomson et al 2007).

\section{Pharmacokinetics during pregnancy}

Maternal plasma concentrations measured during the third trimester were compared to a "baseline" before pregnancy or after delivery in two small studies (Tomson et al 2007;
Westin et al 2008). Both studies found plasma concentrations to be significantly lower during the third trimester in comparison with baseline. The mean concentration-to-dose ratio in the third trimester was $50 \%-30 \%$ of that at baseline. This suggested that the elimination of LEV may be enhanced during pregnancy. However, there was great variability between patients, such that the change in serum concentration could not be accurately predicted.

\section{Serum levels}

LEV has linear kinetics, such that in any individual the serum concentration is proportional to the dose (Patsalos 2004). However, the effective serum level for LEV is not known. One study in 69 patients taking 500-3000 mg/day found that the trough plasma concentration ranged from 1.1 to $33.5 \mu \mathrm{g} / \mathrm{mL}$ (Lancelin et al 2007). Similar mean concentrations were found in patients experiencing adverse effects and those without adverse effects (11.2 vs $10.9 \mu \mathrm{g} / \mathrm{mL})$. The mean plasma concentrations in responders and nonresponders were 12.9 and $9.5 \mu \mathrm{g} / \mathrm{mL}$. The difference was not significant, but the authors suggested that $11 \mu \mathrm{g} / \mathrm{mL}$ could be a threshold concentration for a therapeutic response. The vast majority of patients in this study had refractory epilepsy, making it difficult to study the effective plasma concentration of LEV. Such a study is best conducted in patients with new onset epilepsy. A trial comparing LEV and carbamazepine in newly diagnosed patients did not report plasma concentrations (Brodie et al 2007). However, it found that most patients were seizure-free at the lowest LEV dose of $1000 \mathrm{mg} /$ day. In the therapeutic drug monitoring study mentioned earlier, a daily dose of $1000 \mathrm{mg} /$ day was associated with a mean trough level of $6.5 \pm 2.4 \mu \mathrm{g} / \mathrm{mL}$ (Lancelin et al 2007). Even though a therapeutic and toxic LEV concentration are not defined, measuring the serum concentration is helpful to assess compliance. In addition, if a baseline serum concentration is obtained during a period of good seizure control, the serum concentration can be repeated with breakthrough seizures to assess if a drop in concentration played a role. Finally, monitoring serum concentration through the course of pregnancy can help with calculating the recommended dose adjustments needed to correct for increased clearance.

\section{Putative mechanism of action}

LEV is different in its mechanism from that of other AEDs, because it is not effective in the standard animal models used to screen for anticonvulsant activity, while it is effective in the chronic kindling model (Loscher and Honack 1993; Klitgaard et al 1998). It was recently established that the 
most relevant LEV mechanism of action is through binding to the synaptic vesicle protein SV2A (Lynch et al 2004). The SV2A binding affinity of LEV derivatives correlated strongly with their binding affinity in the brain, as well as with their ability to protect against seizures in the audiogenic mouse model (Lynch et al 2004). Similar findings were noted in the mouse corneal kindling model and the GAERS rat model of generalized absence epilepsy (Kaminski et al 2008). The specific effect of LEV binding to SV2A appears to be a reduction in the rate of vesicle release (Yang et al 2007). LEV has other mechanisms of action that likely play a comparatively smaller role: reversing the inhibition of neuronal GABA- and glycine-gated currents by the negative allosteric modulators zinc and B-carbolines (Rigo et al 2002), and partial depression of the N calcium current (Niespodziany et al 2001; Lukyanetz et al 2002). At present, the mechanisms of action have not yet helped identify a specific clinical efficacy profile for LEV.

\section{Levetiracetam efficacy - pivotal double- blinded randomized controlled trials} Adjunctive therapy in refractory partial epilepsy in adults

LEV was found efficacious in 3 pivotal placebo-controlled randomized blinded clinical trials in adults with refractory partial epilepsy. These trials investigated three doses, 1000, 2000, and $3000 \mathrm{mg} /$ day. All three doses were found to be effective. The US trial compared $1000 \mathrm{mg} /$ day and $3000 \mathrm{mg} /$ day (in two divided doses) with placebo (Cereghino et al 2000). The study randomized 294 patients, 268 of whom completed the 14 weeks of treatment. After a 12-week singleblind baseline, LEV was titrated over 4 weeks. Patients in the $1000 \mathrm{mg}$ /day group first received $333 \mathrm{mg} /$ day for 2 weeks, then $666 \mathrm{mg} /$ day for 2 weeks, while patients in the $3000 \mathrm{mg} /$ day group received $1000 \mathrm{mg} /$ day for 2 weeks and then $2000 \mathrm{mg} /$ day for 2 weeks. The median percentage reduction in seizures over baseline was $32.5 \%$ for LEV $1000 \mathrm{mg} /$ day and $37.1 \%$ for LEV $3000 \mathrm{mg}$ /day as compared with $6.8 \%$ for placebo. The $50 \%$ responder rates were $33 \%$ for $1000 \mathrm{mg} /$ day and $39.8 \%$ for $3000 \mathrm{mg} /$ day, compared with $10.8 \%$ for placebo. Seizure freedom was noted in $3 \%$ of patients in the $1000 \mathrm{mg}$ group and $8 \%$ of the $3000 \mathrm{mg}$ group. No patients were seizure-free in the placebo group. Maximum efficacy was already present in the first visit 2 weeks after initiating titration.

The European placebo-controlled randomized doubleblind trial compared $2000 \mathrm{mg} /$ day, $1000 \mathrm{mg}$ /day, and placebo as add-on treatment (Shorvon et al 2000). Patients randomized to $2000 \mathrm{mg} /$ day received $500 \mathrm{mg}$ bid for 2 weeks, then
$1000 \mathrm{mg}$ bid while patients randomized to $1000 \mathrm{mg} /$ day received placebo for 2 weeks, then $500 \mathrm{mg}$ bid. The 4-week titration period was followed by a 12 -week maintenance phase. Out of 324 randomized patients, 278 completed the study. There was a $26.5 \%$ median seizure reduction from baseline for the $2000 \mathrm{mg} /$ day group, $17.7 \%$ for the 1000 $\mathrm{mg} /$ day group, and $6.1 \%$ for the placebo group. The $50 \%$ responder rate was $31.6 \%$ for the $2000 \mathrm{mg}$ /day group, $22.8 \%$ for the $1000 \mathrm{mg} /$ day group, and $10.4 \%$ for the placebo group. Two percent of the $2000 \mathrm{mg}$ patients, $5 \%$ of the 1000 mg patients, and $1 \%$ of the $112 \mathrm{mg}$ placebo patients were seizure free. In both the US and European trials, both doses tested were more efficacious than the placebo, but were not significantly different from each other.

A third pivotal trial, also conducted in Europe, only compared $3000 \mathrm{mg}$ per day to a placebo (Ben-Menachem and Falter 2000). After the baseline phase, patients randomized to $\mathrm{LEV}$ received $1000 \mathrm{mg} /$ day for 2 weeks, then $2000 \mathrm{mg} /$ day for 2 weeks before receiving $3000 \mathrm{mg} /$ day for the remainder of the trial. The median reduction in seizure frequency from baseline was $39.9 \%$ for LEV compared with $7.2 \%$ for placebo. The responder rate was $50 \%$ for LEV compared with $16.7 \%$ for placebo. Seizure freedom was reported in $8.2 \%$ of LEV patients compared with $1 \%$ of placebo patients.

The findings from the above trials were confirmed in a smaller blinded trial (94 patients) conducted in Taiwan, comparing adjunctive $2000 \mathrm{mg}$ /day of LEV to placebo (Tsai et al 2006). The responder rate in the LEV group was 53.5\% compared with $10.6 \%$ in the placebo group. Seizure freedom was observed in $8.7 \%$ of LEV patients, but none of the placebo patients.

The three main pivotal trials received a number of post hoc analyses. Two of these analyses addressed the latency for onset of action of LEV. In one study, it was found that the increase in proportion of seizure-free patients over baseline was $15 \%$ for the first day of treatment and $17 \%$ for second and third days of treatment for $1000 \mathrm{mg} /$ day, all statistically significant (French and Arrigo 2005). However the increases for $333 \mathrm{mg} /$ day were $7 \%$ for Day 1 and $9 \%$ for the second and third days. These were not significant. There were no major changes in the placebo group. In a second analysis, the mean proportion of seizure-free days were as computed during each week after initiation of treatment (French et al 2005). The mean proportion of seizure-free days was greater in the LEV than the placebo group and the difference was observed as early as the first week after initiation of treatment. Interestingly, it was also greatest at that point in time, after which it dropped but remained fairly stable. A similar 
observation was made in the Taiwanese study, with initial $69 \%$ reduction in seizure frequency at the 2 -week visit after starting LEV, compared with only $37.5 \%$ reduction at the end of the study (Tsai et al 2006).

Another post hoc analysis addressed the number of seizure-free days (Leppik et al 2003a). Addition of LEV increased the number of days without seizures by 5.19 per quarter. An additional analysis addressed the affect of LEV on subtypes of partial seizures in the pooled data from the three major pivotal trials (Leppik et al 2003b). A statistically significant reduction in the frequency of all partial seizures subtypes was observed. In addition, there was an independent reduction of secondarily generalized seizures over and above the reduction of partial seizures.

\section{Add-on treatment for refractory partial seizures in children}

One pediatric double-blind, placebo-controlled, randomized trial was performed in the US (Glauser et al 2006), in which 216 patients were randomized, but 198 patients provided evaluable data. The target dose of LEV was $60 \mathrm{mg} / \mathrm{kg} /$ day in 2 divided doses. Patients first received $20 \mathrm{mg} / \mathrm{kg} /$ day for 2 weeks, then $40 \mathrm{mg} / \mathrm{kg} /$ day for 2 weeks before reaching the final target dose. Patients unable to tolerate $60 \mathrm{mg} / \mathrm{kg} / \mathrm{day}$ could be reduced to $40 \mathrm{mg} / \mathrm{kg} /$ day. The median percentage seizure reduction from baseline was $43.8 \%$ for LEV compared with $23.3 \%$ for placebo. For the whole treatment period, the median reduction was $43.3 \%$ for LEV compared with $16.3 \%$ for placebo. The $50 \%$ responder rate was $44.6 \%$ for LEV and $19.6 \%$ for placebo. The above results were all statistically significant in favor of LEV. Seizure freedom was reported in $6.9 \%$ of LEV patients compared to $1 \%$ of placebo patients.

\section{Monotherapy in new onset epilepsy}

LEV was compared to controlled release carbamazepine in patients with newly diagnosed epilepsy in a double-blind trial (Brodie et al 2007). Patients enrolled in the study were adults with 2 or more partial or generalized tonic-clonic seizures in the previous year. The initial dose assigned was either LEV $500 \mathrm{mg}$ twice a day or controlled-release carbamazepine (CBZ-CR) $200 \mathrm{mg}$ twice a day. The dose could then be increased if a seizure occurred within 26 weeks of stabilization, with a maximum of $1,500 \mathrm{mg}$ bid of LEV or $600 \mathrm{mg}$ bid of CBZ-CR. Patients who were seizure free for 6 months continued on treatment for another 6 months. The intent to treat population included 285 patients randomly assigned to LEV and 291 patients assigned to CBZ-CR.
The per protocol population (no major protocol deviations affecting efficacy) included 237 and 235 patients at 6 months and 228 and 224 patients at 1 year for LEV and CBZ-CR. At 6 months, $73 \%$ of LEV and $72.8 \%$ of CBZ-CR patients were seizure free, and at 1 year $56.6 \%$ of LEV and $58.5 \%$ of CBZCR patients were seizure free, based on the per protocol population. Withdrawal rates for adverse events were $14.4 \%$ with LEV and 19.2\% with CBZ-CR, based on the intent to treat population. The difference was not significant.

Approximately $80 \%$ of patients experienced at least one adverse event in both groups. There was not much difference between the two groups with respect to the adverse events reported, except that more patients in the LEV group reported depression and insomnia while more patients in the CBZ$\mathrm{CR}$ group reported back pain. This study was unique among comparative newly diagnosed epilepsy trials in that it used a controlled-release preparation of carbamazepine. It also had a flexibility in dosing that gave each agent the best chances of success with limited adverse experiences. The lowest dose levels produced seizure freedom at 6 months in the majority of patients in both groups $(59.1 \%$ of LEV patients and $62.1 \%$ of CBZ-CR patients). Thus, $80.1 \%$ of LEV patients who were seizure free at 6 months did become seizure free at the starting dose (Brodie et al 2007).

This adequately powered study showed that LEV was not inferior to CBZ-CR in the treatment of newly diagnosed patients with epilepsy. Based on the results LEV was granted an indication for monotherapy in newly diagnosed patients in the European Union. However, this trial did not satisfy US Federal Drug Administration (FDA) requirements for monotherapy indications.

\section{Adjunctive therapy in patients with idiopathic generalized epilepsy and generalized tonic-clonic seizures}

LEV was compared with placebo as add-on therapy in a double-blind study in patients with idiopathic generalized epilepsy (Berkovic et al 2007). Patients were required to have at least 3 generalized tonic-clonic seizures during an 8-week (4-week retrospective and 4-week prospective) baseline. The study allowed enrollment of patients aged 4-65 years. However, only about $10 \%$ of patients were under 16 years of age. Patients were receiving one or two baseline antiepileptic drugs. The dose of LEV used was $3,000 \mathrm{mg} /$ day or $60 \mathrm{mg} / \mathrm{kg} /$ day for children younger than 16 years and weighing less than $50 \mathrm{~kg}$. At the end of the base-

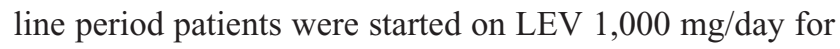
2 weeks, then $2,000 \mathrm{mg} /$ day for 2 weeks, then $3,000 \mathrm{mg} /$ day. 
The corresponding doses for children were $20 \mathrm{mg} / \mathrm{kg} /$ day, 40 $\mathrm{mg} / \mathrm{kg} /$ day, then $60 \mathrm{mg} / \mathrm{kg} /$ day. Patients unable to tolerate the final target dose were allowed to reduce their dose back to the previous value of $2,000 \mathrm{mg} / \mathrm{day}$ or $40 \mathrm{mg} / \mathrm{kg} / \mathrm{day}$. The primary efficacy parameter was reduction in generalized tonic-clonic seizure frequency from baseline. A total of 164 patients were randomized, 80 to $\mathrm{LEV}$, and 84 to placebo. In each group 70 patients completed the evaluation. The primary efficacy variable was significant in favor of the LEV-treated group: the mean percentage reduction in weekly frequency was $56.5 \%$ for LEV and $28.2 \%$ for placebo $(p=0.004)$, and the median percentage reduction was $77.6 \%$ for LEV and $44.6 \%$ for placebo $(\mathrm{p}<0.001)$. The $50 \%$ responder rate was $72.2 \%$ for LEV and $45.2 \%$ for placebo ( $p<0.001$ ). As previously noted in the add-on trials for partial epilepsy, there was a rapid onset of action with $64.6 \%$ of patients classified as responders at the lowest dose of $1,000 \mathrm{mg} /$ day. There was no evidence of seizure exacerbation; fewer patients in the LEV than in the placebo group experienced a $25 \%$ or greater increase in GTC frequency. The percentage of GTC seizurefree patients was $34.2 \%$ in the LEV and $10.7 \%$ in the placebo groups ( $\mathrm{p}<0.001)$. A slightly smaller percentage of patients were free of all seizure types ( 24.1 vs $8.3 \% ; p=2.009$ ). LEV was well tolerated in this trial, with only $1.3 \%$ of LEV and $4.8 \%$ of placebo patients discontinuing treatment due to an adverse experience. The proportion of patients with at least one adverse experience was comparable in the two groups. Fatigue, somnolence, headache, and irritability were the only adverse experiences considered drug-related and reported in more than $5 \%$ of patients. This trial earned LEV approval for adjunctive therapy in the treatment of generalized tonicclonic seizures in idiopathic generalized epilepsy.

\section{Adjunctive therapy in patients with refractory myoclonic seizures}

LEV was recently studied in a double-blind multicenter randomized placebo-controlled study trial in adolescents and adults with idiopathic generalized epilepsy with myoclonic seizures (Noachtar et al 2008). Patients had to be 12 years or older and had to be experiencing at least 8 days with myoclonic seizures during the 8 -week baseline period. The study design included a single-blind baseline period of 8 weeks, a 4-week titration period, and a 12 -week maintenance period. Patients were started on 1,000 mg/day of LEV for 2 weeks, then $2,000 \mathrm{mg} /$ day for 2 weeks, then $3,000 \mathrm{mg} /$ day for the maintenance period. Patients unable to tolerate this dose were allowed to reduce their dose to the previous level of $2,000 \mathrm{mg} /$ day. The primary efficacy endpoint was the responder rate with respect to the number of days with myoclonic seizures. Of the 122 patients randomized, the vast majority had a diagnosis of juvenile myoclonic epilepsy. Sixty patients receiving LEV and 60 receiving placebo could be analyzed. Among LEV patients, 58.3\% had at least 50\% reduction in myoclonic seizure days, compared with $23.3 \%$ of placebo patients $(\mathrm{p}<0.001)$. Very similar results were obtained when evaluating days with any seizure type. Freedom from myoclonic seizures was reported in $16.7 \%$ of LEV patients compared with only $3.3 \%$ of placebo patients during the whole treatment period and in $25 \%$ of LEV patients and $5 \%$ of placebo patients during the 12 -week evaluation period $(\mathrm{p}<0.005)$. When assessing freedom from all seizures, $13.3 \%$ of LEV and $0 \%$ of placebo patients were seizure free during the whole treatment period and $21.7 \%$ of LEV and $1.7 \%$ of placebo patients were seizure free during the 12 -week evaluation period ( $\mathrm{p}<0.001$ ). This study earned LEV approval as adjunctive therapy in patients with myoclonic seizures. In fact, LEV is the only new antiepileptic drug with such approval.

The above study reported generalized tonic-clonic seizures in $41 \mathrm{LEV}$ and 40 placebo patients, and absence seizures in $29 \mathrm{LEV}$ and 21 placebo patients. However, the study was not designed to evaluate these seizure types. No worsening was reported for either seizure type.

\section{Levetiracetam efficacy in specific patient subsets or specific syndromes}

LEV was studied in several non-blinded trials in specific patient populations. Some of these will be briefly reviewed.

\section{Subsets of partial epilepsy}

No blinded randomized trial specifically evaluated subsets of partial epilepsy based on localization or on prior epilepsy surgery. However, one study reported that patients with temporal lobe epilepsy were more likely to be improved than patients with frontal lobe epilepsy (Bazil et al 2002). In another study, patients who had failed epilepsy surgery were much more likely to respond to LEV than patients who had never been operated: $76.1 \%$ were responders and $47.6 \%$ became seizure free compared with $34.3 \%$ responder rate and $14.7 \%$ seizure free rate in patients who never had epilepsy surgery (Motamedi et al 2003). In a study evaluating long-term outcome in patients who initially failed epilepsy surgery, newly administered LEV was a significant positive prognostic factor for eventual seizure remission (Janszky et al 2005). 


\section{LEV efficacy in community patients}

A large open-label trial in 1,030 community patients in the US (KEEPER trial) demonstrated that adjunctive LEV therapy was more effective than noted in double-blind trials in highly refractory patients; $57.9 \%$ of patients were responders and 20\% were seizure free (Morrell et al 2003). Another community-based trial conducted internationally (SKATE trial) also demonstrated higher responder and seizure-free rates than in the double-blind trials (Steinhoff et al 2005; Genton et al 2006; Lambrechts et al 2006).

\section{Efficacy in the elderly}

A subset of patients from the KEEPER trial aged 65 and older were analyzed separately (Ferrendelli et al 2003). They had a better response than the group as a whole. $76.9 \%$ were responders and $40 \%$ were seizure free during the 16 weeks of the study. Another study found a better response to LEV in late-onset epilepsy (Bazil et al 2002).

\section{Benign epilepsy of childhood with centrotemporal spikes (BECTS)}

Children with BECTS do not always need to be treated because they may have a small number of seizures and the eventual outcome is favorable. However, treatment is often initiated in children with young age at onset, frequent seizures, and daytime secondarily generalized seizures. The ability to use an antiepileptic drug with favorable tolerability would make the decision to treat easier and less controversial. LEV was compared with oxcarbazepine (OXC) as monotherapy in children with newly diagnosed BECTS (Coppola et al 2007). The patients were aged 3-12 years, had a clear diagnosis of BECTS, a normal MRI, no neurological deficits, and a reason for treatment such as frequent seizures. Children were randomized for treatment with either LEV or OXC. Both drugs were initiated at $5 \mathrm{mg} / \mathrm{kg} /$ day followed by a 3-day titration at increments of $5 \mathrm{mg} / \mathrm{kg}$ up to a maximum daily dose of $20 \mathrm{mg} / \mathrm{kg}$. The titration stopped earlier if the medication was not tolerated. In case of seizure recurrence, the LEV dose could be increased up to $30 \mathrm{mg} / \mathrm{kg}$ and the OXC dose up to $35 \mathrm{mg} / \mathrm{kg}$. Twenty-one children were randomized to LEV and 18 to OXC. After follow-up for 12-24 months (mean 18.5 months), $90.5 \%$ of LEV and $72.2 \%$ of OXC patients had no seizure recurrence. Adverse effects of LEV were reported in 3 children (14.3\%) and OXC in 2 children (11.1\%). The trial suggested that both LEV and OXC could be used in patients with BECTS. Favorable efficacy and tolerability of LEV in BECTS was also suggested in extended case reports (Bello-Espinosa and Roberts 2003; Verrotti et al 2007).

\section{Severe myoclonic epilepsy of infancy (SMEI)}

SMEI is a rare epileptic syndrome usually related to a sodium channel mutation. The syndrome is characterized by highly refractory seizures (including generalized myoclonic, generalized tonic-clonic, generalized absence, and partial seizures), and slowing or arrest of psychomotor development. There is a strong need for effective therapy in this syndrome. One adjunctive open-label multi-center trial conducted in Italy enrolled patients who had failed at least 2 AEDs and had at least 4 generalized tonic-clonic seizures during an 8-week historical baseline (Striano et al 2007). LEV was titrated up to $50-60 \mathrm{mg} / \mathrm{kg} / \mathrm{day}$. Twenty-three of 28 enrolled patients (82.1\%) completed the 18-week trial. There was a significant reduction in the weekly frequency of generalized tonic-clonic, myoclonic, and partial seizures, but not absence seizures. The responder rate was $64.2 \%$ for tonic-clonic seizures, $60 \%$ for myoclonic seizures, $60 \%$ for focal seizures, and $44 \%$ for absence seizures. Seizure freedom for specific seizure types was recorded in 2-3 patients for each of generalized tonic-clonic, generalized myoclonic, and focal seizures, but it was not clear how many patients were free of all seizures. An excellent response of SMEI to LEV was also reported in another study, with 3 out of 4 patients classified as responders (Labate et al 2006). Thus LEV appeared particularly useful for this difficult type of epilepsy.

\section{Progressive myoclonic epilepsies}

Progressive myoclonic epilepsy includes several specific conditions in which myoclonus, both epileptic and nonepileptic, is a prominent manifestation. Seizures and myoclonus tend to be refractory, and new effective therapies are needed. LEV efficacy was demonstrated in various progressive myoclonic epilepsy syndromes, mainly for the myoclonus. In one study of 13 patients with Unverricht Lundborg disease, 8 had a measurable improvement in myoclonus score, with the best effect in younger patients (Magaudda et al 2004). Marked improvement in myoclonus and quality of life was also reported in smaller groups (Kinrions et al 2003; Papacostas et al 2007). One other study reported benefit in 3 of 6 patients with Unverricht-Lundborg disease and 2 of 3 with myoclonic epilepsy with ragged red fibers (MERRF). However, there was long-term attenuation of initial efficacy in 3 of the patients (Crest et al 2004). There was also 1 single case report of dramatic improvement in 1 patient with MERRF (Mancuso et al 2006), and a report of LEV benefit in 2 sisters with Lafora body disease (Boccella et al 2003). Of note is that there have been several reports of LEV in non-epileptic myoclonus, with variable success (Frucht et al 2001; Genton 
and Gelisse 2001; Krauss et al 2001; Keswani et al 2002; Schauer et al 2002; Imperiale et al 2003; Lim and Ahmed 2005; Striano et al 2005).

\section{Lennox-Gastaut and West syndrome}

In patients with Lennox-Gastaut syndrome, a good response of myoclonic and tonic-clonic seizures was reported (De Los Reyes et al 2004). There was also some response of atonic seizures, but tonic seizures were not responsive to treatment (De Los Reyes et al 2004). There are rare reports of LEV efficacy in West syndrome (Lagae et al 2003; Lawlor and Devlin 2005). One study included a variety of epileptic syndromes, including West syndrome and Lennox-Gastaut syndrome (Lagae et al 2003). Approximately $50 \%$ of patients were responders, with no significant differences in efficacy between generalized and partial epilepsy syndromes. The best results were observed for myoclonic seizures with 64\% responders (Lagae et al 2003).

\section{Other epileptic syndromes with myoclonic seizures}

Several case series and open label trials investigated LEV use in epileptic syndromes that included myoclonic seizures. LEV efficacy was most strongly demonstrated in juvenile myoclonic epilepsy, both as add-on therapy and monotherapy (Krauss et al 2003; Kumar and Smith 2004; Di Bonaventura et al 2005; Labate et al 2006; Specchio et al 2006; Sharpe et al 2008). Particular benefit was noted in previously drug-naïve patients. These reports complement the pivotal trials in juvenile myoclonic epilepsy, and also suggest that LEV could be considered for investigation as first-line therapy in this condition. Anecdotal benefit was reported in myoclonic astatic epilepsy, myoclonic absences, and benign myoclonic epilepsy in infancy (Labate et al 2006), and a dramatic improvement was reported in one patient with negative myoclonus (Gelisse et al 2003).

\section{Epilepsy with continuous spikes and waves during slow wave sleep and Landau-Kleffner syndrome}

One report of LEV use in 12 patients with continuous spikes and waves during slow sleep indicated that 7 patients $(58.3 \%)$ showed improvement of the EEG, with 3 of them showing improvement on neuropsychological evaluation. Two other patients without improvement of the EEG had neuropsychological improvement (Aeby et al 2005). Benefit was also reported in another small series (Capovilla et al 2004). There was 1 case report of improvement in a child with the related Laudau-Kleffner syndrome (Kossoff et al 2003).

\section{Photosensitive epilepsy}

LEV abolished or suppressed the photoparoxysmal response on EEG in 9 of 12 patients with generalized photosensitive epilepsy, in a short-term trial designed to explore LEV effect in photosensitivity (Kasteleijn-Nolst Trenite et al 1996). There were no specific therapeutic trials in photosensitive epilepsy, but 1 paper reported excellent benefit from LEV in one patient with refractory photosensitive epilepsy (Lagae et al 2003).

\section{Generalized absence seizures}

There have been no specific studies of LEV for generalized absence seizures. In the pivotal study of LEV in patients with myoclonic seizures, there was no significant difference between LEV and placebo in reduction of seizure days with absence seizures (Andermann et al 2005). In another study of idiopathic generalized epilepsy, absence seizures were the least likely to respond to LEV (Krauss et al 2003). However, it should be noted that refractory absence is not necessarily representative of generalized absence seizures in general.

\section{Status epilepticus and acute repetitive seizures}

The rapid almost complete absorption of LEV and its rapid onset of action made it a candidate for use in acute seizure conditions. A number of case reports and case series have supported a role for oral LEV in the treatment of acute repetitive seizures and status epilepticus that were refractory to initial therapy (Rossetti and Bromfield 2005; Zaatreh 2005; Patel et al 2006; Rossetti and Bromfield 2006; Rupprecht et al 2007; Trabacca et al 2007; Alehan et al 2008). The largest study included included 23 patients with status epilepticus, 10 of whom responded (Rossetti and Bromfield 2006). The study found that response was more likely when LEV is administered early, and that additional benefit was unlikely when the dose is escalated beyond $3000 \mathrm{mg} /$ day. Since the advent of intravenous LEV, there have been several reports of successful use of intravenous LEV in the treatment of status epilepticus or acute repetitive seizures that had failed benzodiazepines or phenytoin ( Falip et al 2006; Farooq et al 2007; Knake et al 2007; Schulze-Bonhage et al 2007; Abend et al 2008; Goraya et al 2008; Ruegg et al 2008). The largest report described the result of treatment of 18 episodes of focal status epilepticus in 16 patients (Knake et al 2007). All had failed benzodiazepine therapy. The loading LEV dose varied from 250 to $1500 \mathrm{mg}$, followed by daily maintenance of 1500-7000 mg. Status epilepticus resolved after intravenous LEV in 16 episodes, and only 2 episodes required additional therapy (Knake et al 2007). No severe adverse experiences were reported in these studies, and LEV was usually welltolerated. These encouraging reports support the need for studies comparing LEV with other established therapies for various types of status epilepticus. 


\section{Prediction of LEV efficacy}

One study in 344 patients attempted to identify factors that predicted response to therapy (Kinirons et al 2006). In this study, $16.3 \%$ of patients were seizure free and $42 \%$ were responders. Idiopathic generalized epilepsy and post-traumatic partial epilepsy were more common in the responder group $(p=0.005$ and $p=0.05)$. In responders lamotrigine was used significantly more often in combination with LEV ( $p=0.003)$. Not unexpectedly, the mean daily LEV dose was lower in responders than non-responders.

\section{Paradoxical increase in seizures}

A paradoxical increase in seizures was noted at times, usually at elevated doses. In one study that focused on the paradoxical increase in seizures $40 \%$ of adults and $20 \%$ of children were responders, but $18 \%$ of adults and $43 \%$ of children had a greater than $25 \%$ increase in seizure frequency (Nakken et al 2003). The increase in seizure frequency was more likely in mentally retarded patients and at relatively high doses. One other study showed that worsening of seizures was generally seen early in the titration phase (Coppola et al 2004).

\section{Long-term maintenance of efficacy}

The report of development of tolerance in one animal model of epilepsy (Loscher and Honack 2000) prompted evaluation of LEV long-term efficacy in patients with epilepsy. Some of the long-term studies analyzed the trial data and others analyzed post marketing data. In the analysis of the trial data base, the continuation rate was $60 \%$ after 1 year (Krakow et al 2001). Factors that predicted continuation of LEV were a high maximal dose, a low starting dose, the presence of generalized tonic-clonic seizures, and a smaller number of AEDs at baseline. Thirteen per cent of the patients became seizure free for at least 6 months and $8 \%$ for at least 1 year; $4.5 \%$ of patients became seizure free from the first day of exposure until the cut-off point. The total trial population was divided into cohorts based on the duration of exposure (6-month increments) and the median percentage reduction in seizure frequency was examined for each of the cohorts (Ben-Menachem et al 2003). Overall, the median percentage reduction was $39.6 \%$ and there was no decline in that parameter within each cohort. In fact, the median percent reduction appeared to increase rather than decrease over time. During the last 6 months of treatment $11.7 \%$ of patients were seizure free overall. The stability of response was also evaluated by examining the percentage of responders in the first 3 months who remained responders in the subsequent 3 months and the percentage of the latter who remained responders for the next 3 months (Abou-Khalil and Lazenby 2003). The analysis indicated that $73.6 \%$ of the 3 -month responders remained responders for the next three months and $82 \%$ of these were still responders in the subsequent 3 months. Thus, LEV response appears to be maintained for the majority of patients, but a small percentage of individuals may have a reduction in benefit while others may have an improvement.

The post-marketing studies had a similar conclusion (Abou-Khalil and Lazenby 2003; Betts et al 2003; Ben-Menachem and Gilland 2003; Nicolson et al 2004; Depondt et al 2006; Kuba et al 2006). The retention rates at 1 year varied from $61 \%$ to $77 \%$ and seizure freedom rate varied from $16 \%$ to $26 \%$. In one study, there was a slight reduction in seizure freedom from $32 \%$ at 6 months to $26 \%$ at 1 year (Betts et al 2003); among patients who were seizure free at 6 months, $74 \%$ were still seizure free at 1 year and $18 \%$ were still more than $90 \%$ improved, though no longer seizure free. Another study with follow-up for 1 to 2 years, $81.5 \%$ of patients who were seizure free in the first 3 months were still seizure free in the last 3 months of treatment, but 39\% of those who were seizure free in the last 3 months were not seizure free in the first 3 months (Abou-Khalil and Lazenby 2003). The phenomenon of AED tolerance may possibly be playing a role in a small proportion of patients treated with LEV. This phenomenon is recognized with other antiepileptic drugs as well, but its degree is not clearly understood (Loscher and Schmidt 2006).

\section{Levetiracetam tolerability}

The initial placebo-controlled adjunctive trials in partial epilepsy suggested that treatment emergent adverse events that had a higher frequency with LEV were somnolence, asthenia, dizziness, and infection (upper respiratory infections). Somnolence was the most common reason for LEV discontinuation in the US pivotal partial seizure trial (Cereghino et al 2000). Its frequency ranged from $5 \%$ to $20 \%$ in the adult pivotal trials (Ben-Menachem and Falter 2000; Cereghino et al 2000; Shorvon et al 2000; Berkovic et al 2007; Brodie et al 2007; Noachtar et al 2008; ), and 23\% in the pediatric trial (Glauser et al 2006). The most common adverse events did not seem dose related in the studies that evaluated more than one LEV dose (Cereghino et al 2000; Shorvon et al 2000). However, in one study comparing 2,000 and 4,000 mg/day without titration, somnolence was highest in patients receiving 4,000 mg/day, affecting 44.7\% (Betts et al 2000). Adverse effects generally appeared within the first month of treatment. In one trial somnolence was reported in $10 \%$ of patients during LEV up-titration, but not in the evaluation period (Noachtar 
et al 2008). Drowsiness seems more common in older age. In one post-marketing study comparing 151 younger adults (age 16-31 years) and 157 older adults (age 55-88 years), drowsiness was reported by $12 \%$ of the younger and $24.7 \%$ of the older group. It resulted in dose reduction or discontinuation in 6.9 and $3.1 \%$ of the younger group and $15.1 \%$ and $5.2 \%$ of the older group.

Behavioral/psychiatric adverse effects were not prominent in the initial adjunctive therapy trials in partial epilepsy. However, these were more prominent in subsequent trials performed in less refractory patients (AbouKhalil et al 2003). In a systematic review of LEV safety in the clinical trial population, non-psychotic behavioral symptoms occurred in $13.5 \%$ of LEV-treated patients with epilepsy versus $6 \%$ of placebo-treated patients (French et al 2001). The difference between placebo and LEV groups was small or non-existent in cognitive and anxiety studies, suggesting that behavioral adverse effects may be specific for epilepsy. It is also possible that the greater occurrence in epilepsy patients could be contributed to by higher dose in the epilepsy group. The non-psychotic behavioral symptoms that occurred in more than $1 \%$ of epilepsy patients in placebo-controlled trials were depression $(3.8 \%)$, nervousness $(3.8 \%)$, hostility $(2.3 \%)$, anxiety (1.8\%), and emotional lability (1.7\%) (Cramer et al 2003). The proportion of patients with these symptoms was somewhat higher in an open-label study that included less refractory patients (Abou-Khalil et al 2003). Among 219 patients in that study nervousness was reported in $9.6 \%$, depression in $7.3 \%$, hostility in $4.1 \%$, personality disorder in $3.7 \%$, emotional lability in $2.7 \%$, and anxiety in $2.3 \%$ of subjects (Abou-Khalil et al 2003). Behavioral adverse events were severe in 7 of 219 patients (3.2\%).

Behavioral adverse events may be more likely in certain patient groups. Learning disabilities were a predisposing factor in one study that reported a $23 \%$ frequency of behavioral adverse effects when learning disability was present compared to $10 \%$ if learning disability was absent (Brodtkorb et al 2004). One other study indicated a greater frequency of behavioral adverse effects in patients with previous psychiatric history, history of febrile convulsions, and a history of status epilepticus, and a lower frequency when lamotrigine was used concomitantly with LEV (Mula et al 2003, 2004). Similar risk factors were noted for psychiatric adverse effects with topiramate, suggesting that a subgroup of patients is generally prone to develop these adverse effects during AED therapy, independent of AED mechanism of action (Mula et al 2007).
Behavioral adverse events are most often mild and are not usually a cause of LEV discontinuation. However, behavioral adverse effects had a greater representation in patients who discontinued LEV, and may be the most common reason for discontinuation (Abou-Khalil and Lazenby 2003). In one large case controlled study, more than half of the patients who discontinued LEV did so because of behavioral issues, the most important of which were depression, irritability, and aggression (White et al 2003). Patients who discontinued LEV because of behavioral adverse events were more likely to have symptomatic generalized epilepsy, history of psychiatric diagnosis, and a faster LEV titration (White et al 2003). Behavioral adverse experiences were reported with a higher frequency in pediatric studies. In the pivotal pediatric adjunctive placebo-controlled trial, 5 behavioral/psychiatric adverse effects were reported in $>5 \%$ of patients: hostility (12\% of LEV and $6 \%$ of placebo), nervousness (10\% of LEV vs $2 \%$ of placebo), personality disorder ( $8 \%$ of LEV vs $7 \%$ of placebo), emotional lability ( $6 \%$ of LEV vs $4 \%$ of placebo), and agitation ( $6 \%$ of LEV, $1 \%$ of placebo). None of these adverse events were seen in $>5 \%$ of patients in the adult adjunctive partial epilepsy trials. In the adjunctive generalized epilepsy trial, irritability occurred in $6.3 \%$ and mood swings in $5.1 \%$ of LEV-treated patients versus $2.4 \%$ and $1.2 \%$ of placebo-treated patients (Berkovic et al 2007). However, in the myoclonic seizure trial nervousness was reported in $3.3 \%$ of LEV-treated and $6.7 \%$ of placebo patients. In the newly diagnosed epilepsy trial, the main behavioral adverse effect reported was depression in $6.3 \%$ of LEV patients and $2.1 \%$ of carbamazepine-treated patients (Brodie et al 2007).

Behavioral adverse effects were reported more often in pediatric than adult case series (Glauser et al 2002; Wheless and Ng 2002; De Los Reyes et al 2004). However, improvements in behavior were also common. In one study, $12.8 \%$ of children demonstrated aggression and 10.3\% hyperactivity, but $25.6 \%$ had an improvement in behavior and/or cognition (Wheless and Ng 2002).

Psychosis has been reported rarely with LEV therapy (Kossoff et al 2001; Motamedi et al 2003; Youroukos et al 2003). The symptoms were always reversed with LEV discontinuation. Psychosis may occur rarely with many AEDs, and is unlikely to be specific for LEV.

It is of note that LEV is not associated with serious systemic adverse effects. One common concern, allergic rash is uncommon with LEV. In one large post-marketing study, the risk of rash with LEV use was $0.6 \%$, significantly lower than the average of all AEDs (Arif et al 2007). 


\section{Safety in pregnancy and breast feeding}

Limited data are available on safety in pregnancy. In the analysis of the UK Epilepsy and Pregnancy Registry, 3 of 117 exposed pregnancies had a major congenital malformation, but all three were also exposed to other AEDs (Hunt et al 2006). There were also no minor malformations in the LEV monotherapy group which included 39 monotherapy exposures. Other smaller reports also did not identify any LEV-related malformations (Long 2003; ten Berg et al 2005). Four infants exposed to LEV monotherapy had a low birth rate, but the mean birth weight for infants exposed to LEV was within the normal range (Hunt et al 2006). Thus, preliminary data seemed favorable, but additional reports are needed for definitive assessment of LEV safety during pregnancy.

LEV is extensively transferred from mother into breast milk. However, breast fed infants had very low LEV serum concentrations, suggesting that breastfeeding should not be contraindicated (Johannessen et al 2005; Tomson et al 2007).

\section{Quality of life}

Quality of life measurements were incorporated in the US pivotal LEV trial. The QOLIE-31 questionnaire was administered at the end of the baseline period before randomization and again at the end of the treatment period. It showed improvement in 3 of the 7 items: overall quality of life, seizure worry, and cognitive functioning (Cereghino et al 2000). The results were analyzed in greater detail in a separate publication (Cramer et al 2000). Statistically significant improvements were found in seizure worry and overall quality of life in the LEV treatment group. The placebo group scores decreased for the cognitive functioning subscale and the total score. QOLIE scores were influenced by seizure control. Patients who had a $50 \%$ or greater improvement in seizures had significant improvements in all areas compared with non-responders. The exception was medication effect. Therefore, LEV seemed to have a positive impact on healthrelated quality of life.

One hundred and one patients who completed QOLIE-31 at the end of double-blind treatment also completed the questionnaire during a long-term follow-up visit, approximately 4 years after starting LEV (Cramer and Van Hammee 2003). All scales and the total score improved between the baseline and long-term assessments. The short-term improvement noted at the end of the double-blind treatment period was maintained in the long term, and patients who were randomized to placebo reached the same level of improvement in the long-term as patients who initially received LEV.
One recent study used QOLIE-31 at baseline, at 16 weeks of double-blind add-on treatment, then again at 40 weeks of treatment (now open treatment). Fourteen patients were randomized to LEV and 14 to placebo. Subscale scores on the QOLIE-31, including scores on Cognitive Functioning and Social Function improved only for the LEV group at the end of short-term treatment. At the end of the long-term phase, these improvements were maintained (Zhou et al 2008).

\section{Cognitive function}

LEV cognitive effects were examined in comparison to carbamazepine (CBZ) in 28 healthy volunteers, using a randomized, double-blind, two-period crossover design (Meador et al 2007). The doses were adjusted to mid-range therapeutic level, with a mean of $7.5 \mu \mathrm{g} / \mathrm{mL}$ for $\mathrm{CBZ}$ and $32.2 \mu \mathrm{g} / \mathrm{mL}$ for LEV. CBZ was worse than LEV on 15 of 34 variables tested, and better at none. Compared with baseline, CBZ was worse for 26 of 34 variables, and LEV was worse for 4 (Meador et al 2007).

There is no evidence of decline in cognitive function with LEV treatment in patients with partial epilepsy. One study compared LEV with topiramate (TPM) using standardized a neuropsychological test battery. Testing was performed before treatment and after reaching steady state in 30 consecutive patients with focal epilepsy treated with LEV and 21 treated with TPM. Whereas the TPM group worsened in cognitive speed, verbal fluency, and short-term memory, there was no change in the LEV group (Gomer et al 2007). Another study found that performance time on the Wisconsin Card Sorting Test and Delayed Logic Memory significantly improved for 14 patients randomized to LEV, but not for 14 randomized to placebo (Zhou et al 2008).

\section{Place of levetiracetam in therapy Partial epilepsy}

LEV has not been compared directly to other new AEDs, but meta-analysis of controlled partial epilepsy adjunctive trials suggested that LEV had a favorable 'responder-withdrawal ratio' in comparison with other agents (Marson et al 2001; Otoul et al 2005; Zaccara et al 2006). Based on the favorable efficacy and tolerability of LEV as an add-on therapy in refractory partial epilepsy, it is reasonable to consider it one of the first add-on therapies in these patients. Other factors that argue for this are the absence of drug - drug interactions and the rapid onset of action, which means that it will rapidly become clear whether LEV will be effective. However the choice of therapy is influenced by many factors including co-morbidity. The co-morbidity of obesity, for example, may 
favor adjunctive therapy with an AED that can cause weight loss, for example topiramate or zonisamide. Psychiatric comorbidity may also argue against choosing LEV as initial adjunctive therapy.

The place of LEV as an initial monotherapy is less clear cut. There is now a well designed monotherapy study that showed non-inferiority to controlled-release carbamazepine (Brodie et al 2007), likely to satisfy the criteria of the Therapeutics and Technology Assessment Subcommittee of the American Academy of Neurology and the American Epilepsy Society for initial monotherapy use (French et al 2004). LEV has approval by the European Medicines Agency but not the US FDA for initial monotherapy for partial onset seizures. Despite the absence of US FDA approval, there is ample evidence that LEV is widely used in the hospital setting for new onset epilepsy and acute seizures (Chabolla et al 2003; Glass et al 2005; Falip et al 2006; Di Bonaventura et al 2006; Szaflarski et al 2007). This was the case even before the appearance of the intravenous formulation, but the intravenous formulation certainly has made this use more prevalent. The other antiepileptic drugs with intravenous formulations have associated disadvantages. Phenobarbital is highly sedating and both phenobarbital and phenytoin/fosphenytoin are enzyme inducing and could result in important unfavorable interactions. Intravenous valproate is safe and well tolerated, but potential adverse effects with long-term valproate use may be a deterrent to its use in hospitalized patients. As a result of the above, LEV has become frequently used in patients with seizures secondary to stroke, neurosurgical intervention, brain tumors, and other medical conditions (Chabolla et al 2003; Wagner et al 2003; Glass et al 2005; Di Bonaventura et al 2006; Falip et al 2006; Newton et al 2006, 2007; Maschio et al 2006; Szaflarski et al 2007).

The IV formulation of LEV is currently approved for temporary replacement in patients who cannot take oral medication (Ramael et al 2006a, b; Baulac et al 2007). It is not approved for the treatment of status epilepticus. However, there are now several reports of the use of LEV (usually oral LEV) in refractory status epilepticus (Atefy and Tettenborn 2005; Rossetti and Bromfield 2005; Patel et al 2006; Rossetti and Bromfield 2006; Rupprecht et al 2007; Schulze-Bonhage et al 2007), and it is certainly reasonable to consider IV LEV as one of the options for patients with non-convulsive partial status epilepticus as well as refractory status epilepticus, particularly in those patients who have recurrence of status in conjunction with anesthesia withdrawal. LEV may also play a role for patients who have to be started or restarted on LEV abruptly. The intravenous administration of LEV is associated with a peak level at the end of infusion in 5 or 15 minutes, whereas the oral administration is associated with a peak at 1 hour, and the peak would be delayed if administration is with food (Ramael et al 2006a, b).

\section{Generalized epilepsy}

There is now definite evidence of LEV efficacy as adjunctive therapy for patients with idiopathic generalized epilepsy and uncontrolled generalized tonic-clonic seizures or generalized myoclonic seizures (Berkovic et al 2007; Noachtar et al 2008). LEV can therefore be considered as an early adjunctive therapy in these conditions. In the case of myoclonic seizures, it is the only new antiepileptic drug with an FDA approved indication for this application (one older antiepileptic drug, clonazepam, also has FDA approval for myoclonic seizures).

LEV has no FDA indication and no pivotal trials supporting initial monotherapy use in generalized epilepsy. However, the evidence of adjunctive efficacy in juvenile myoclonic epilepsy has prompted use as initial monotherapy in juvenile myoclonic epilepsy. The anecdotal data has been very favorable (Specchio et al 2006; Sharpe et al 2008). However, the use of LEV as initial monotherapy cannot be strongly supported without a pivotal trial, for example a trial comparing it with valproate. In women of childbearing potential, valproate use is associated with unacceptable risks including teratogenicity in the event of pregnancy, weight gain, hair loss, and hormonal changes. As a result of these risks, it has become common in practice to use alternatives such as lamotrigine, topiramate, and zonisamide (Prasad et al 2003). None of these drugs have pivotal trials supporting their use for juvenile myoclonic epilepsy. LEV could be added to that list, with the advantage of definitive evidence for efficacy as add-on therapy. The one seizure type for which there is no data is absence seizures. Based on lack of data, LEV would not be an appropriate initial therapy for absence seizures or an early adjunctive agent for refractory absence seizures.

\section{Status epilepticus}

Intravenous LEV may be considered in the treatment of nonconvulsive or focal status epilepticus refractory to initial therapy. In this setting, the risk of general anesthesia may outweigh the risk of neuronal injury from ongoing seizure activity, such that additional nonsedating antiepileptic therapy may be used. The dose of intravenous LEV should probably be 1000-1500 mg administered over 5 minutes. Generalized convulsive status epilepticus should be treated 
with standard intravenous therapy (usually lorazepam followed by fosphenytoin or phenytoin). General anesthesia should be considered next if initial therapy is not effective. LEV may be used only if it can be added without delaying standard therapy or general anesthesia when standard therapy fails.

\section{Dosing recommendations}

The prescribing information recommends an adult starting dose of $1000 \mathrm{mg} /$ day (500 mg twice daily), with subsequent escalation by $1000 \mathrm{mg}$ every 2 weeks up to $1500 \mathrm{mg}$ twice daily. These recommendations are based on the dose used in pivotal trials. Using evidence from post-marketing analyses, the author favors a smaller starting dose of $250 \mathrm{mg}$ twice daily, with escalation to $500 \mathrm{mg}$ twice daily after 1 week if the starting dose is well tolerated. In patients at higher risk for behavioral - psychiatric adverse effects, the starting dose can even be smaller, at $250 \mathrm{mg}$ at bedtime. The same approach can be used with the elderly who have a higher chance of experiencing adverse effects on LEV. A starting dose of 500 $\mathrm{mg}$ twice daily can still be considered in hospitalized patients who need faster efficacy. Automatic escalation of the LEV dose to $3000 \mathrm{mg} /$ day is usually not necessary. In patients with infrequent seizures, for whom the minimal effective dose can be hard to determine, the LEV dose can be escalated to $1000 \mathrm{mg}$ twice daily. The dose of LEV can be increased up to $3000 \mathrm{mg} /$ day for persistent seizures. Even though there is no clear benefit beyond $3000 \mathrm{mg} /$ day, the LEV dose can be increased to $4000 \mathrm{mg} /$ day for patients who have clearly responded to LEV, but have residual breakthrough seizures. However, the treating physician has to be aware of the risk of seizure exacerbation at higher LEV doses.

The same dosing guidelines can be applied to children. The official prescribing information recommends starting at $20 \mathrm{mg} / \mathrm{kg} /$ day in 2 divided doses, with subsequent increments of $20 \mathrm{mg} / \mathrm{kg}$ every 2 weeks up to $60 \mathrm{mg} / \mathrm{kg} /$ day. However, starting at $10 \mathrm{mg} / \mathrm{kg}$ may reduce the frequency or intensity of behavioral adverse effects and provide a greater opportunity to manage these adverse effects rather than stop LEV. The target dose should be tailored to the patient, such that escalation to $60 \mathrm{mg} / \mathrm{kg} /$ day may not be necessary.

\section{Summary}

In summary LEV should be considered as an initial or early add-on therapy for partial epilepsy, initial or early add-on therapy for myoclonic seizures in patients with juvenile myoclonic epilepsy, and as an early add-on therapy for patients with generalized tonic-clonic seizures in the setting of idiopathic generalized epilepsy. There is also evidence to support use of LEV as initial monotherapy in partial epilepsy, but other indications are not supported by pivotal trials.

\section{Disclosures}

The author has received research grant support from UCB Pharma and has given occasional lectures sponsored by the UCB Pharma Speakers Bureau.

\section{References}

Abend NS, Florance N, Finkel RS et al. 2008. Intravenous levetiracetam terminates refractory focal status epilepticus. Neurocrit Care. Jan 8 [Epub ahead of print].

Abou-Khalil B, Hemdal P, Privitera MD. 2003. An open-label study of levetiracetam at individualised doses between 1000 and $3000 \mathrm{mg}$ day-1. in adult patients with refractory epilepsy. Seizure, 12:141-9.

Abou-Khalil B, Lazenby B. 2003. Long-term experience with levetiracetam. Epileptic Disord, 5(Suppl 1):S33-7.

Aeby A, Poznanski N, Verheulpen D, et al. 2005. Levetiracetam efficacy in epileptic syndromes with continuous spikes and waves during slow sleep: experience in 12 cases. Epilepsia, 46:1937-42.

Alehan F, Ozcay F, Haberal M. 2008. The use of levetiracetam in a child with nonconvulsive status epilepticus. J Child Neurol, 23:331-3.

Allegaert K, Lewi L, Naulaers G, et al. 2006. Levetiracetam pharmacokinetics in neonates at birth. Epilepsia, 47:1068-9.

Andermann E, Andermann F, Meyvisch P, et al. 2005. Seizure control with levetiracetam in juvenile myoclonic epilepsies. Epilepsia, 46(Suppl 8):205.

Arif H, Buchsbaum R, Weintraub D, et al. 2007. Comparison and predictors of rash associated with 15 antiepileptic drugs. Neurology, 68:1701-9.

Atefy R, Tettenborn B. 2005. Nonconvulsive status epilepticus on treatment with levetiracetam. Epilepsy Behav, 6:613-16.

Baulac M, Brodie MJ, Elger CE, et al. 2007. Levetiracetam intravenous infusion as an alternative to oral dosing in patients with partial-onset seizures. Epilepsia, 48:589-92.

Bazil CW, Rose A, Resor S, et al. 2002. Levetiracetam may be more effective for late-onset partial epilepsy. Arch Neurol, 59:1905-8.

Beghi E Gatti G, Tonini C, et al. 2003. Adjunctive therapy versus alternative monotherapy in patients with partial epilepsy failing on a single drug: a multicentre, randomised, pragmatic controlled trial. Epilepsy Res, 57:1-13.

Bello-Espinosa LE, Roberts SL. 2003. Levetiracetam for benign epilepsy of childhood with centrotemporal spikes-three cases. Seizure, 12:157-9.

Ben-Menachem E, Edrich P, Van Vleymen B, et al. 2003. Evidence for sustained efficacy of levetiracetam as add-on epilepsy therapy. Epilepsy Res, 53:57-64.

Ben-Menachem E, Falter U. 2000. Efficacy and tolerability of levetiracetam $3000 \mathrm{mg} / \mathrm{d}$ in patients with refractory partial seizures: a multicenter, double-blind, responder-selected study evaluating monotherapy. European Levetiracetam Study Group. Epilepsia, 41:276-83.

Ben-Menachem E, Gilland E. 2003. Efficacy and tolerability of levetiracetam during 1-year follow-up in patients with refractory epilepsy. Seizure, 12:31-5.

Berkovic SF, Knowlton RC, Leroy RF, et al. 2007. Placebo-controlled study of levetiracetam in idiopathic generalized epilepsy. Neurology, 69:1751-60.

Betts T, Waegemans T, Crawford P. 2000. A multicentre, double-blind, randomized, parallel group study to evaluate the tolerability and efficacy of two oral doses of levetiracetam, $2000 \mathrm{mg}$ daily and $4000 \mathrm{mg}$ daily, without titration in patients with refractory epilepsy. Seizure, 9:0-7.

Betts T, Yarrow H, Greenhill L, et al. 2003. Clinical experience of marketed Levetiracetam in an epilepsy clinic-a one year follow up study. Seizure, 12:36-40. 
Boccella P, Striano P, Zara F, et al. 2003. Bioptically demonstrated Lafora disease without EPM2A mutation: a clinical and neurophysiological study of two sisters. Clin Neurol Neurosurg, 106:5-9.

Brodie MJ, Perucca E, Ryvlin P, et al. 2007. Comparison of levetiracetam and controlled-release carbamazepine in newly diagnosed epilepsy. Neurology, 68:402-8.

Brodtkorb E, Klees TM, Nakken KO, et al. 2004. Levetiracetam in adult patients with and without learning disability: focus on behavioral adverse effects. Epilepsy Behav, 5:31-5.

Browne TR, Szabo GK, Leppik IE, et al. 2000. Absence of pharmacokinetic drug interaction of levetiracetam with phenytoin in patients with epilepsy determined by new technique. J Clin Pharmacol, 40:90-5.

Capovilla G, Beccaria F, Cagdas S, et al. 2004. Efficacy of levetiracetam in pharmacoresistant continuous spikes and waves during slow sleep. Acta Neurol Scand, 110:44-7.

Cereghino JJ, Biton V, Abou-Khalil B, et al. 2000. Levetiracetam for partial seizures: results of a double-blind, randomized clinical trial. Neurology, 55:36-42.

Chabolla DR, Harnois DM, Meschia JF. 2003. Levetiracetam monotherapy for liver transplant patients with seizures. Transplant Proc, 35:480-1.

Commission. 1981. Proposal for revised clinical and electroencephalographic classification of epileptic seizures. From the Commission on Classification and Terminology of the International League Against Epilepsy. Epilepsia, 22:89-501.

Commission 1989. Proposal for revised classification of epilepsies and epileptic syndromes. Commission on Classification and Terminology of the International League Against Epilepsy. Epilepsia, 30:389-99.

Coppola G, Franzoni E, Verrotti A, et al. 2007. Levetiracetam or oxcarbazepine as monotherapy in newly diagnosed benign epilepsy of childhood with centrotemporal spikes BECTS: an open-label, parallel group trial. Brain Dev, 29:81-4.

Coppola G, Mangano S, Tortorella G, et al. 2004. Levetiracetam during 1 -year follow-up in children, adolescents, and young adults with refractory epilepsy. Epilepsy Res, 59:5-42.

Coupez R, Nicolas JM, Browne TR. 2003. Levetiracetam, a new antiepileptic agent: lack of in vitro and in vivo pharmacokinetic interaction with valproic acid. Epilepsia, 44:71-8.

Cramer JA, Arrigo C, Van Hammee G, et al. 2000. Effect of levetiracetam on epilepsy-related quality of life. N132 Study Group. Epilepsia, 41:68-74.

Cramer JA, De Rue K, Devinsky O, et al. 2003. A systematic review of the behavioral effects of levetiracetam in adults with epilepsy, cognitive disorders, or an anxiety disorder during clinical trials. Epilepsy Behav, 4:24-32.

Cramer JA, Van Hammee G. 2003. Maintenance of improvement in healthrelated quality of life during long-term treatment with levetiracetam. Epilepsy Behav, 4:118-23.

Crest C, Dupont S, Leguern E, et al. 2004. Levetiracetam in progressive myoclonic epilepsy: an exploratory study in 9 patients. Neurology, 62:40-3.

De Los Reyes EC, Sharp GB, Williams JP, et al. 2004. Levetiracetam in the treatment of Lennox-Gastaut syndrome. Pediatr Neurol, 30:54-6.

Depondt C, Yuen AW, Bell GS, et al. 2006. The long term retention of levetiracetam in a large cohort of patients with epilepsy. $J$ Neurol Neurosurg Psychiatry, 77:01-3.

Di Bonaventura C, Fattouch J, Mari F, et al. 2005. Clinical experience with levetiracetam in idiopathic generalized epilepsy according to different syndrome subtypes. Epileptic Disord, 7:31-5.

Di Bonaventura C, Mari F, Fattouch J, et al. 2006. Use of levetiracetam in treating epilepsy associated with other medical conditions. Acta Neurol Scand, 113:2-6.

Engel J Jr. 2001. A proposed diagnostic scheme for people with epileptic seizures and with epilepsy: report of the ILAE Task Force on Classification and Terminology. Epilepsia, 42:96-803.

Falip M, Carreno M, Amaro S, et al. 2006. Use of levetiracetam in hospitalized patients. Epilepsia, 47:186-8.
Farooq MU, Naravetla B, Majid A, et al. 2007. IV levetiracetam in the management of non-convulsive status epilepticus. Neurocrit Care, 7:36-9.

Ferrendelli JA, French J, Leppik I, et al. 2003. Use of levetiracetam in a population of patients aged 65 years and older: a subset analysis of the KEEPER trial. Epilepsy Behav, 4:02-9.

French J. 2001. Use of levetiracetam in special populations. Epilepsia, 42(Suppl 4):40-3.

French J, Arrigo C. 2005. Rapid onset of action of levetiracetam in refractory epilepsy patients. Epilepsia, 46:324-6.

French J, di Nicola S, Arrigo C. 2005. Fast and sustained efficacy of levetiracetam during titration and the first 3 months of treatment in refractory epilepsy. Epilepsia, 46:1304-7.

French J, Edrich P, Cramer JA. 2001. A systematic review of the safety profile of levetiracetam: a new antiepileptic drug. Epilepsy Res, 47:77-90.

French JA, Kanner AM, Bautista J et al. 2004. Efficacy and tolerability of the new antiepileptic drugs II: treatment of refractory epilepsy: report of the Therapeutics and Technology Assessment Subcommittee and Quality Standards Subcommittee of the American Academy of Neurology and the American Epilepsy Society. Neurology, 62:1261-73.

Frucht SJ, Louis ED, Chuang C, et al. 2001. A pilot tolerability and efficacy study of levetiracetam in patients with chronic myoclonus. Neurology, 57:1112-4.

Gelisse P, Crespel A, Genton P, et al. 2003. Dramatic effect of levetiracetam on epileptic negative myoclonus. Acta Neurol Scand, 107:302-3.

Genton P, Gelisse P. 2001. Suppression of post-hypoxic and post-encephalitic myoclonus with levetiracetam. Neurology, 57:1144-5.

Genton P, Sadzot B, Fejerman N, et al. 2006. Levetiracetam in a broad population of patients with refractory epilepsy: interim results of the international SKATE trial. Acta Neurol Scand, 113:387-94.

Glass GA, Stankiewicz J, Mithoefer A, et al. 2005. Levetiracetam for seizures after liver transplantation. Neurology, 64:1084-5.

Glauser TA, Ayala R, Elterman RD, et al. 2006. Double-blind placebocontrolled trial of adjunctive levetiracetam in pediatric partial seizures. Neurology, 66:1654-60.

Glauser TA, Mitchell WG, Weinstock A, et al. 2007. Pharmacokinetics of levetiracetam in infants and young children with epilepsy. Epilepsia, 48:1117-42.

Glauser TA, Pellock JM, Bebin EM, et al. 2002. Efficacy and safety of levetiracetam in children with partial seizures: an open-label trial. Epilepsia, 43:518-24.

Gomer B, Wagner K, Frings L, et al. 2007. The influence of antiepileptic drugs on cognition: a comparison of levetiracetam with topiramate. Epilepsy Behav, 10:486-94.

Goraya JS, Khurana DS, Valencia I, et al. 2008. Intravenous Levetiracetam in Children With Epilepsy. Pediatr Neurol, 38:177-80.

Hirsch LJ, Arif H, Buchsbaum R,et al. 2007. Effect of age and comedication on levetiracetam pharmacokinetics and tolerability. Epilepsia, 48:1351-9.

Hunt S, Craig J, Russell A, et al. 2006. Levetiracetam in pregnancy: preliminary experience from the UK Epilepsy and Pregnancy Register. Neurology, 67:1876-9.

Imperiale D, Bortolotto S, Cucatto A, et al. 2003. Levetiracetam control of myoclonus in a patient with Creutzfeldt-Jakob disease. Eur Neurol, 49:189-90.

Janszky J, Pannek HW, Janszky I, et al. 2005. Failed surgery for temporal lobe epilepsy: Predictors of long-term seizure-free course. Epilepsy Res, 64:35-44.

Johannessen SI, Helde G, Brodtkorb E. 2005. Levetiracetam concentrations in serum and in breast milk at birth and during lactation. Epilepsia, 46:775-7.

Kaminski RM, Matagne A, Leclercq K, et al. 2008. SV2A protein is a broad-spectrum anticonvulsant target: Functional correlation between protein binding and seizure protection in models of both partial and generalized epilepsy. Neuropharmacology, 54:715-20. 
Kasteleijn-Nolst Trenite DG, Marescaux C, Stodieck S, et al. 1996. Photosensitive epilepsy: a model to study the effects of antiepileptic drugs. Evaluation of the piracetam analogue, levetiracetam. Epilepsy Res, 25:225-30.

Keswani SC, Kossoff EH, Krauss GL, et al. 2002. Amelioration of spinal myoclonus with levetiracetam. J Neurol Neurosurg Psychiatry, 73:457-8.

Kinirons P, McCarthy M, Doherty CP, et al. 2006. Predicting drug-resistant patients who respond to add-on therapy with levetiracetam. Seizure, 15:387-92.

Kinrions P, Ibrahim N, Murphy K, et al. 2003. Efficacy of levetiracetam in a patient with Unverricht-Lundborg progressive myoclonic epilepsy. Neurology, 60:1394-5.

Klitgaard H, Matagne A, Gobert J, et al. 1998. Evidence for a unique profile of levetiracetam in rodent models of seizures and epilepsy. Eur $J$ Pharmacol, 353:191-206.

Knake S, Gruener J, Hattemer K, et al. 2007. Intravenous levetiracetam in the treatment of benzodiazepine-refractory status epilepticus. J Neurol Neurosurg Psychiatry. Sep 26 [Epub ahead of print].

Kossoff EH, Bergey GK, Freeman JM, et al. 2001. Levetiracetam psychosis in children with epilepsy. Epilepsia, 42:1611-3.

Kossoff EH, Boatman D, Freeman JM. 2003. Landau-Kleffner syndrome responsive to levetiracetam. Epilepsy Behav, 4:571-5.

Krakow K, Walker M, Otoul C, et al. 2001. Long-term continuation of levetiracetam in patients with refractory epilepsy. Neurology, 56:1772-4.

Krauss GL, Bergin A, Kramer RE, et al. 2001. Suppression of post-hypoxic and post-encephalitic myoclonus with levetiracetam. Neurology, 56:411-2.

Krauss GL, Betts T, Abou-Khalil B, et al. 2003. Levetiracetam treatment of idiopathic generalised epilepsy. Seizure, 12:617-20.

Kuba R, Novotna I, Brazdil M, et al. 2006. Long-term levetiracetam treatment of epilepsy patients: clinical audit. Epilepsy Res, 72:111-9.

Kumar SP, Smith PE. 2004. Levetiracetam as add-on therapy in generalised epilepsies. Seizure, 13:475-7.

Kwan P, Brodie MJ. 2000a. Early identification of refractory epilepsy. N Engl J Med, 342:314-9.

Kwan P, Brodie MJ. 2000b. Epilepsy after the first drug fails: substitution or add-on? Seizure, 9:464-8.

Labate A, Colosimo E, Gambardella A, et al. 2006. Levetiracetam in patients with generalised epilepsy and myoclonic seizures: an open label study. Seizure, 15:214-8.

Lagae L, Buyse G, Deconinck A, et al. 2003. Effect of levetiracetam in refractory childhood epilepsy syndromes. Eur J Paediatr Neurol, 7:123-8.

Lambrechts DA, Sadzot B, van Paesschen W, et al. 2006. Efficacy and safety of levetiracetam in clinical practice: results of the SKATE trial from Belgium and The Netherlands. Seizure, 15:434-42.

Lancelin F, Franchon E, Kraoul L, et al. 2007. Therapeutic drug monitoring of levetiracetam by high-performance liquid chromatography with photodiode array ultraviolet detection: preliminary observations on correlation between plasma concentration and clinical response in patients with refractory epilepsy. Ther Drug Monit, 29:576-83.

Lawlor KM, Devlin AM. 2005. Levetiracetam in the treatment of infantile spasms. Eur J Paediatr Neurol, 9:19-22.

Leppik I, Morrell M, Godfroid P, et al. 2003a. Seizure-free days observed in randomized placebo-controlled add-on trials with levetiracetam in partial epilepsy. Epilepsia, 44:1350-2.

Leppik IE, Biton V, Sander JW, et al. 2003b. Levetiracetam and partial seizure subtypes: pooled data from three randomized, placebo-controlled trials. Epilepsia, 44:1585-7.

Levy RH, Ragueneau-Majlessi I, Baltes E. 2001. Repeated administration of the novel antiepileptic agent levetiracetam does not alter digoxin pharmacokinetics and pharmacodynamics in healthy volunteers. Epilepsy Res, 46:93-9.

Lim LL, Ahmed A. 2005. Limited efficacy of levetiracetam on myoclonus of different etiologies. Parkinsonism Relat Disord, 11, 135-7.
Long L. 2003. Levetiracetam monotherapy during pregnancy: a case series. Epilepsy Behav, 4:447-8.

Loscher W, Honack D. 1993. Profile of ucb L059, a novel anticonvulsant drug, in models of partial and generalized epilepsy in mice and rats. Eur J Pharmacol, 232:147-58.

Loscher W, Honack D. 2000. Development of tolerance during chronic treatment of kindled rats with the novel antiepileptic drug levetiracetam. Epilepsia, 41:1499-506.

Loscher W, Schmidt D. 2006. Experimental and clinical evidence for loss of effect (tolerance) during prolonged treatment with antiepileptic drugs. Epilepsia, 47:1253-84.

Lukyanetz EA, Shkryl VM, Kostyuk PG. 2002. Selective blockade of N-type calcium channels by levetiracetam. Epilepsia, 43:9-18.

Lynch BA, Lambeng N, Nocka K, et al. 2004. The synaptic vesicle protein SV2A is the binding site for the antiepileptic drug levetiracetam. Proc Natl Acad Sci USA, 101:9861-6.

Magaudda A, Gelisse P, Genton P. 2004. Antimyoclonic effect of levetiracetam in 13 patients with Unverricht-Lundborg disease: clinical observations. Epilepsia, 45:678-81

Mancuso M, Galli R, Pizzanelli C, et al. 2006. Antimyoclonic effect of levetiracetam in MERRF syndrome. J Neurol Sci, 243:97-9.

Marson AG, Hutton JL, Leach JP, et al. 2001. Levetiracetam, oxcarbazepine, remacemide and zonisamide for drug resistant localization-related epilepsy: a systematic review. Epilepsy Res, 46:259-70.

Maschio M, Albani F, Baruzzi A, et al. 2006. Levetiracetam therapy in patients with brain tumour and epilepsy. J Neurooncol, 80:97-100.

May TW, Rambeck B, Jurgens U. 2003. Serum concentrations of Levetiracetam in epileptic patients: the influence of dose and co-medication. Ther Drug Monit, 25:690-9.

Meador KJ, Gevins A, Loring DW, et al. 2007. Neuropsychological and neurophysiologic effects of carbamazepine and levetiracetam. Neurology, 69:2076-84.

Morrell MJ, Leppik I, French J, et al. 2003. The KEEPER trial: levetiracetam adjunctive treatment of partial-onset seizures in an open-label community-based study. Epilepsy Res, 54:153-61.

Motamedi M, Nguyen DK, Zaatreh M, et al. 2003. Levetiracetam efficacy in refractory partial-onset seizures, especially after failed epilepsy surgery. Epilepsia, 44:211-4.

Mula M, Trimble MR, Sander JW. 2004. Psychiatric adverse events in patients with epilepsy and learning disabilities taking levetiracetam. Seizure, 13:55-7.

Mula M, Trimble MR, Sander JW. 2007. Are psychiatric adverse events of antiepileptic drugs a unique entity? A study on topiramate and levetiracetam. Epilepsia, 48:2322-6.

Mula M, Trimble MR, Yuen A, et al. 2003. Psychiatric adverse events during levetiracetam therapy. Neurology, 61:704-6.

Nakken KO, Eriksson AS, Lossius R, et al. 2003. A paradoxical effect of levetiracetam may be seen in both children and adults with refractory epilepsy. Seizure, 12:42-6.

Newton HB, Dalton J, Goldlust S, et al. 2007. Retrospective analysis of the efficacy and tolerability of levetiracetam in patients with metastatic brain tumors. J Neurooncol, 84:293-6.

Newton HB, Goldlust SA, Pearl D. 2006. Retrospective analysis of the efficacy and tolerability of levetiracetam in brain tumor patients. J Neurooncol, 78:99-102.

Nicolas JM, Collart P, Gerin B, et al. 1999. In vitro evaluation of potential drug interactions with levetiracetam, a new antiepileptic agent. Drug Metab Dispos, 27:250-4.

Nicolson A, Lewis SA, Smith DF. 2004. A prospective analysis of the outcome of levetiracetam in clinical practice. Neurology, 63:568-70

Niespodziany I, Klitgaard H, Margineanu DG. 2001. Levetiracetam inhibits the high-voltage-activated $\mathrm{Ca} 2^{+}$. current in pyramidal neurones of rat hippocampal slices. Neurosci Lett, 306:5-8.

Noachtar S, Andermann E, Meyvisch P, et al. 2008. Levetiracetam for the treatment of idiopathic generalized epilepsy with myoclonic seizures. Neurology, 70:607-16. 
Otoul C, Arrigo C, Rijckevorsel KV, et al. 2005. Meta-analysis and indirect comparisons of levetiracetam with other second-generation antiepileptic drugs in partial epilepsy. Clin Neuropharmacol, 28:72-8.

Papacostas S, Kkolou E, Papathanasiou E. 2007. Levetiracetam in three cases of progressive myoclonus epilepsy. Pharm World Sci, 29:164-6.

Patel NC, Landan IR, Levin, J, et al. 2006. The use of levetiracetam in refractory status epilepticus. Seizure, 15:137-41.

Patsalos PN. 2000. Pharmacokinetic profile of levetiracetam: toward ideal characteristics. Pharmacol Ther, 85:77-85.

Patsalos PN. 2003. The pharmacokinetic characteristics of levetiracetam. Methods Find Exp Clin Pharmacol, 25:123-9.

Patsalos PN. 2004. Clinical pharmacokinetics of levetiracetam. Clin Pharmacokinet, 43: 707-24.

Patsalos PN, Perucca E. 2003. Clinically important drug interactions in epilepsy: interactions between antiepileptic drugs and other drugs Lancet Neurol, 2:473-81.

Pellock JM, Glauser TA, Bebin EM, et al. 2001. Pharmacokinetic study of levetiracetam in children. Epilepsia, 42:1574-9.

Perucca E, Gidal BE, Baltes E. 2003. Effects of antiepileptic comedication on levetiracetam pharmacokinetics: a pooled analysis of data from randomized adjunctive therapy trials. Epilepsy Res, 53:47-56.

Prasad A, Kuzniecky RI, Knowlton RC, et al. 2003. Evolving antiepileptic drug treatment in juvenile myoclonic epilepsy. Arch Neurol, 60:1100-5.

Radtke RA. 2001. Pharmacokinetics of levetiracetam. Epilepsia, 42(Suppl 4):24-7.

Ragueneau-Majlessi I, Levy RH, Janik F. 2002. Levetiracetam does not alter the pharmacokinetics of an oral contraceptive in healthy women. Epilepsia, 43:697-702.

Ragueneau-Majlessi I, Levy RH, Meyerhoff C. 2001. Lack of effect of repeated administration of levetiracetam on the pharmacodynamic and pharmacokinetic profiles of warfarin. Epilepsy Res, 47:55-63.

Ramael S, Daoust A, Otoul C, et al. 2006a. Levetiracetam intravenous infusion: a randomized, placebo-controlled safety and pharmacokinetic study. Epilepsia, 47:1128-35.

Ramael S, De Smedt F, Toublanc N, et al. 2006b. Single-dose bioavailability of levetiracetam intravenous infusion relative to oral tablets and multiple-dose pharmacokinetics and tolerability of levetiracetam intravenous infusion compared with placebo in healthy subjects Clin Ther, 28:734-44.

Renganathan R, Delanty N. 2003. Juvenile myoclonic epilepsy: underappreciated and under-diagnosed. Postgrad Med J, 79:78-80.

Rigo JM, Hans G, Nguyen L, et al. 2002. The anti-epileptic drug levetiracetam reverses the inhibition by negative allosteric modulators of neuronal GABA- and glycine-gated currents. Br J Pharmacol, 136:659-72.

Rossetti AO, Bromfield EB. 2005. Levetiracetam in the treatment of status epilepticus in adults: a study of 13 episodes. Eur Neurol, 54:34-8.

Rossetti AO, Bromfield EB. 2006. Determinants of success in the use of oral levetiracetam in status epilepticus. Epilepsy Behav, 8:651-4.

Ruegg S, Naegelin Y, Hardmeier M, et al. 2008. Intravenous levetiracetam: Treatment experience with the first 50 critically ill patients. Epilepsy Behav.

Rupprecht S, Franke K, Fitzek S, et al. 2007. Levetiracetam as a treatment option in non-convulsive status epilepticus. Epilepsy Res, 73:238-44.

Schachter SC. 2007. Currently available antiepileptic drugs. Neurotherapeutics, 4:4-11.

Schauer R, Singer M, Saltuari L, et al. 2002. Suppression of cortical myoclonus by levetiracetam. Mov Disord, 17:411-5.

Schulze-Bonhage A, Hefft S, Oehl B. 2007. Termination of complex partial status epilepticus by intravenous levetiracetam - a case report. J Neurol Neurosurg Psychiatry. Mar 12 [Epub ahead of print].

Selwa LM, Schmidt SL, Malow BA, et al. 2003. Long-term outcome of nonsurgical candidates with medically refractory localization-related epilepsy. Epilepsia, 44:1568-72.
Sharpe DV, Patel AD, Abou-Khalil B, et al. 2008. Levetiracetam monotherapy in juvenile myoclonic epilepsy. Seizure, 17:64-8.

Shorvon SD, Lowenthal A, Janz D, et al. 2000. Multicenter double-blind, randomized, placebo-controlled trial of levetiracetam as add-on therapy in patients with refractory partial seizures. European Levetiracetam Study Group. Epilepsia, 41:1179-86.

Specchio LM, Gambardella A, Giallonardo AT, et al. 2006. Open label, long-term, pragmatic study on levetiracetam in the treatment of juvenile myoclonic epilepsy. Epilepsy Res, 71:32-9.

Stefan H, Wang-Tilz Y, Pauli E, et al. 2006. Onset of action of levetiracetam: a RCT trial using therapeutic intensive seizure analysis TISA. Epilepsia, 47:516-22.

Steinhoff BJ, Trinka E, Wieser HG. 2005. Levetiracetam in patients with refractory epilepsy: results of the SKATE trial in Austria, Germany and Switzerland. Seizure, 14:490-6.

Striano P, Coppola A, Pezzella M, et al. 2007. An open-label trial of levetiracetam in severe myoclonic epilepsy of infancy. Neurology, 69:250-4.

Striano P, Manganelli F, Boccella P, et al. 2005. Levetiracetam in patients with cortical myoclonus: a clinical and electrophysiological study. Mov Disord, 20:1610-4.

Szaflarski JP, Meckler JM, Szaflarski M, et al. 2007. Levetiracetam use in critically ill patients. Neurocrit Care, 7:140-7.

ten Berg K, Samren EB, van Oppen AC, et al. 2005. Levetiracetam use and pregnancy outcome. Reprod Toxicol, 20, 175-8.

Tomson T, Palm R, Kallen K, et al. 2007. Pharmacokinetics of levetiracetam during pregnancy, delivery, in the neonatal period, and lactation. Epilepsia, 48:1111-6.

Trabacca A, Profice P, Costanza MC, et al. 2007. Levetiracetam in nonconvulsive status epilepticus in childhood: a case report. J Child Neurol, 22:639-41.

Tsai JJ, Yen DJ, Hsih MS, et al. 2006. Efficacy and safety of levetiracetam up to $2000 \mathrm{mg} /$ day. in Taiwanese patients with refractory partial seizures: a multicenter, randomized, double-blind, placebo-controlled study. Epilepsia, 47:72-81.

Verrotti A, Coppola G, Manco R, et al. 2007. Levetiracetam monotherapy for children and adolescents with benign rolandic seizures. Seizure, 16:271-5.

Wagner GL, Wilms EB, Van Donselaar CA, et al. 2003. Levetiracetam: preliminary experience in patients with primary brain tumours. Seizure, 12:585-6.

Westin AA, Reimers A, Helde G, et al. 2008. Serum concentration/dose ratio of levetiracetam before, during and after pregnancy. Seizure, 17:192-8.

Wheless JW, Ng YT. 2002. Levetiracetam in refractory pediatric epilepsy J Child Neurol, 17:413-5.

White JR, Walczak TS, Leppik IE, et al. 2003. Discontinuation of levetiracetam because of behavioral side effects: a case-control study Neurology, 61:1218-21.

Wirrell EC. 1998. Benign epilepsy of childhood with centrotemporal spikes. Epilepsia, 39(Suppl 4):S32-41.

Yang XF, Weisenfeld A, Rothman SM. 2007. Prolonged exposure to levetiracetam reveals a presynaptic effect on neurotransmission. Epilepsia, 48-1861-9.

Youroukos S, Lazopoulou D, Michelakou D, et al. 2003. Acute psychosis associated with levetiracetam. Epileptic Disord, 5:117-9.

Zaatreh MM. 2005. Levetiracetam in porphyric status epilepticus: a case report. Clin Neuropharmacol, 28:243-4.

Zaccara G, Messori A, Cincotta M, et al. 2006. Comparison of the efficacy and tolerability of new antiepileptic drugs: what can we learn from long-term studies? Acta Neurol Scand, 114:157-68.

Zhou B, Zhang Q, Tian L, et al. 2008. Effects of levetiracetam as an add-on therapy on cognitive function and quality of life in patients with refractory partial seizures. Epilepsy Behav, 12:305-10. 
